\title{
Nonlinear Predictive Control for the Concentrations Profile Regulation under Unknown Reaction Disturbances in a Fuel Cell Anode Gas Channel
}

\author{
Julio Luna*, Carlos Ocampo-Martinez, Maria Serra \\ Universitat Politècnica de Catalunya. Institut de Robòtica i Informàtica Industrial, CSIC-UPC. C/Llorens i Artigas 4-6. \\ 08028 Barcelona, Spain
}

\begin{abstract}
In this work, a nonlinear model predictive control (NMPC) strategy is proposed to regulate the concentrations of the different gas species inside a Proton Exchange Membrane Fuel Cell (PEMFC) anode gas channel. The purpose of the regulation relies on the rejection of the unmeasurable perturbations that affect the system: the hydrogen reaction and water transport terms. The model of the anode channel is derived from the discretisation of the partial differential equations that define the nonlinear dynamics of the system, taking into account spatial variations along the channel. Forward and backward discretisations of the distributed model are employed to take advantage of the boundary conditions of the problem. A linear observer is designed and implemented to perform output-feedback control of the plant. This information is fed to the controller to regulate the states towards their desired values. Simulation results are presented to show the performance of the proposed control method over a given case study. Different cost functions are compared and the one with minimum state-regulation error is identified. Suitable dynamic responses are obtained facing the different considered disturbances.
\end{abstract}

Keywords: Concentration regulation, distributed model, NMPC, fuel cell control, fuel cell observation

\section{Introduction}

Nowadays, fuel cells are an interesting alternative for clean energy production. Particularly, proton exchange membrane fuel cells (PEMFC), with a high power density, are very promising for mass market applications such as automotive and stationary combined heat and power (CHP) systems. The fuel for PEMFC systems is hydrogen, an energy vector that can be obtained via electrolysis or reforming through the consumption of electrical energy. Currently, researchers from all over the world are dedicating a great effort to improve efficiency, reduce degradation and decrease the production costs of PEMFC technology. In the automatic control field, new estimation, diagnosis and control systems are being developed with the same goal in mind.

Therefore, there is already an important number of research works focusing on the automatic control of PEMFC. Different control objectives and techniques can be found in the literature: stoichiometry, minimising input flow rates, temperature and water management are among the most frequent control objectives $[1,2]$; linear controllers, unfalsified controllers [3], predictive controllers, variable structure controllers are some of the used control techniques $[4,5]$. In order to continue exploiting the control engineering potential to improve PEMFC performance, distributed parameter models have acquired increasing importance $[6,7]$. This is due to the increasing concern about the effects of the variation of certain variables along the system. This work is based on a distributed model of the anode channel of a PEMFC, proposing as a control objective to regulate the gas concentration profiles along the channel towards constant set-point profiles.

As any real system, PEMFC is plenty of dynamical behaviours and variables bounded by physical limits that should be considered when designing a control law, e.g., voltages, currents, flows. Moreover, the interaction of the diverse compositional sub-systems determines the definition of several operational constraints that, in the same way as the variable bounds, should be taken into account when formulating a closed-loop control scheme. In this sense, model predictive control (MPC) has been recognized as a powerful methodology since it has the intrinsic ability to deal with system constraints in a systematic and straightforward manner [8]. Added to this fact, there exists other strong reasons for utilizing this control technique such as the capability of considering several variables (multi-variable systems) and control objectives (multi-objective control) as well as the inclusion of system disturbances handling in on-line mode.

MPC is sensitive to the model accuracy since the 
control computation is precisely based on a mathematical model of the plant (in this case, the fuel cell). Moreover, the MPC controller design depends on the nature of the PEMFC model: from the purely nonlinear MPC (NMPC) [9], to linear approaches [10, 11], piece-wise affine (PWA) models $[12,13]$ and hybrid systems forms [14].

The NMPC approach has several advantages due to the consideration of the nonlinear dynamics of the system, key aspect when driving the system far away from its nominal working point (a common situation in PEMFC-based energy systems). On the other hand, one of the main problems that can be encountered when using this control strategy is the high computational burden.

The main contribution of this paper relies on the implementation of a NMPC strategy based on a nonlinear distributed parameters model of an PEMFC anode channel in such a way that the proposed regulation of the anode gases concentrations can be reached by considering an accurate model and a controller that takes into account the physical and operational system constraints. In a previous work [15], a similar controller was implemented assuming statefeedback control and measured disturbances. Both assumptions were highly unrealistic considering the system, but allowed to develop the first approach of the NMPC strategy. This paper presents a solution making use of a state observer to perform outputfeedback control, since it is virtually impossible to know the values of some internal states of a PEMFC. Moreover, the reaction rates and water transport in the system are included in the control approach as unmeasurable disturbances, being the robustness feature of the overall implementation another contribution of this work.

The remainder of the paper is organized as follows. In Section 2, the general system description and statement of the control problem are presented. In Section 3, the mathematical model of the considered system, based on distributed parameters, is developed and explained in detail. In Section 4, the NMPC strategy is stated and implemented based on the model of the anode gas channel developed in Section 3. In Section 5, simulation results are presented and analysed in detail. Finally, in Section 6, the conclusions of this work are presented and some research lines for future work are proposed.

\section{System Description}

This paper is focused on the anode channel of a single-channel PEMFC. The concentration profiles in the anode channel are the controlled variables. The spatial profiles are defined along the z-axis, while the hydrogen and water transport terms, considered as

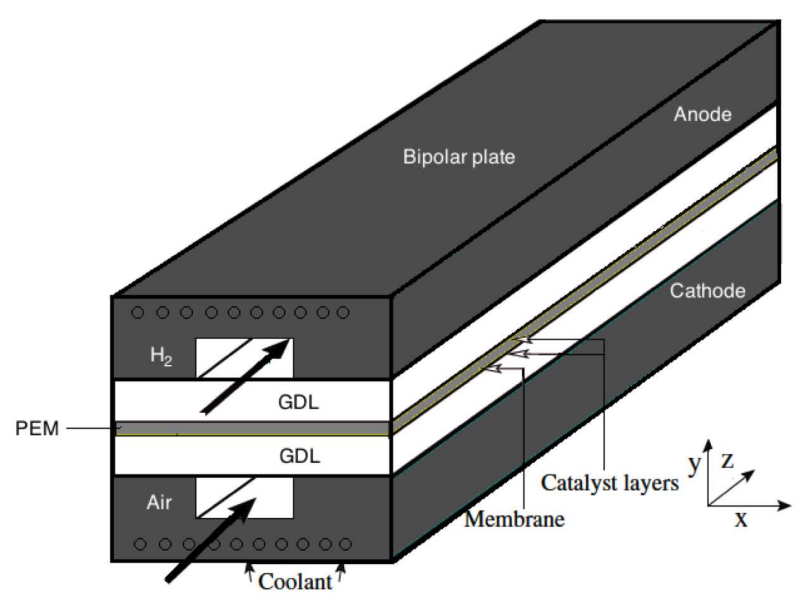

Figure 1: Single-channel PEMFC representation

unknown disturbances, are perpendicular to the supply channel (along the y-axis direction). See Figure 1 for the overview of the PEMFC and the defined reference frame. The nature and computation of these disturbances depend on the rest of the PEMFC model [7] that is not included in this paper since both the reaction and water transpor terms are considered as unknown by the NMPC controller.

The reaction rate at which the hydrogen is consumed depends on the power demanded by the load connected to the PEMFC-based system. Therefore, in order to maintain the hydrogen concentration levels at a certain value during the operation of the system, the hydrogen inflow should be controlled. On the other side, a key aspect to take into account when analysing the efficiency of the PEMFC is the hydration of both the catalyst layer and the membrane. Due to this fact, a second control objective should be considered: the regulation of the water-vapour concentration along the anode channel.

Regarding the water flux, while this paper is focused on the anode-channel side of a PEMFC, it is considered that the water flows through the entire system, being a key element for the electrical conductivity of the PEMFC. The water behaviour at the electrolyte membrane is of special interest due to the key role of the water content in the dynamics of the proton transport, and thus, over the electrical conductivity. It is assumed that the water transport terms take place in the y-axis domain of the system depicted in Figure 1 driven by chemical potential gradients [16]. Moreover, it is also assumed that the membrane can retain water internally [7], the swelling of the membrane is not considered.

The anode gas channel model [7] employed to simulate the control solutions has a z-axis length $(L)$ of $0.4 \mathrm{~m}$ and a $\mathrm{y}$-axis channel thickness $(\delta)$ of $0.7 \mathrm{~mm}$. A general description of the complete single-channel 
PEMFC model can be found in [7], where its dimensions are detailed to be $10 \times 4 \times 400 \mathrm{~mm}$, ignoring the thickness of the bipolar plates.

\section{Mathematical Modelling}

In order to implement the NMPC controller (or any model-based method) to perform the regulation of the plant, a mathematical model that represent the internal dynamics of the system is needed beforehand. In this section, a control-oriented mathematical model of the PEMFC anode gas channel is derived from the partial differential equations that describe the behaviour of the overall mass balances, the flow velocities and the pressure drops along the z-axis of the channel, as developed in [7]. Hence,

$$
\begin{aligned}
\frac{\partial c_{i}(t)}{\partial t} & =-\frac{\partial v(t)}{\partial z} c_{i}(t)-\frac{\dot{n}_{i}(t)}{\delta} \\
v(t) & =-K \frac{\partial p(t)}{\partial z} \\
p(t) & =R T \sum_{i} c_{i}(t)
\end{aligned}
$$

where table 1 collects and defines the variables and parameters involved in the model (1). Hydrogen and water vapour z-dependant concentrations along the anode gas channel are denoted as $c_{i}$, where subscript $i$ stands for the reactant, being $i=H_{2}$ for hydrogen and $i=\mathrm{H}_{2} \mathrm{O}$ for water.

Table 1: Nomenclature and units of variables and parameters in (1)

\begin{tabular}{lll}
\hline parameter & description & units \\
\hline$c_{i}$ & concentration of $i$-th gas & $\mathrm{mol} \mathrm{m}^{-3}$ \\
$\delta$ & thickness of the channel & $\mathrm{m}$ \\
$K$ & pressure drop coefficient & $\mathrm{m}^{2} \mathrm{~s}^{-1} \mathrm{~Pa}^{-1}$ \\
$\dot{n}_{i}$ & molar flux of $i$-th gas & $\mathrm{mol} \mathrm{m}^{-2} \mathrm{~s}^{-1}$ \\
$p$ & pressure & $\mathrm{Pa}$ \\
$R$ & gas constant & $\mathrm{J} \mathrm{mol} \mathrm{K}^{-1}$ \\
$T$ & temperature & $\mathrm{K}$ \\
$v$ & flow velocity & $\mathrm{m} \mathrm{s}^{-1}$ \\
\hline
\end{tabular}

The system has the input molar fluxes and external ambient pressure as known boundary conditions. Knowing this, the spatial discretisation of (1) reduces the complexity of solving analytically the partial differential equations. Therefore, a forward-backward discretisation will be applied to the spatial partial differential equations that define the gas-channel dynamics. As explained before, $\mathrm{H}_{2}$ reaction and water transport terms take place in the $\mathrm{y}$-axis domain of the
PEMFC model. These terms are defined as lumped parameters, being variable along the channel.

The study proposed in this paper can be generalised to the case of $n$ discretised finite-element volumes along the gas channel $\left(n_{\text {disc }}\right)$. In Figure 2, the main structure of the system for this generalisation is presented.

The finite-element discretisation of the partial differential equations in (1) along the z-direction yields the following system:

$$
\begin{aligned}
\dot{c}_{i, j}(t) & =\frac{v_{j-1}(t) c_{i, j-1}(t)}{\Delta z}-\frac{v_{j}(t) c_{i, j}(t)}{\Delta z}-\frac{\dot{n}_{i, j}(t)}{\delta}, \\
v_{j}(t) & =\frac{K}{\Delta z}\left(p_{j}(t)-p_{j+1}(t)\right) \\
p_{j}(t) & =R T \sum_{i} c_{i, j}(t),
\end{aligned}
$$

where the new subscript $j$ is referred to the discretised volume (e.g., $c_{\mathrm{H}_{2} \mathrm{O}, 3}$ is the $\mathrm{H}_{2} \mathrm{O}$ concentration value at the third volume of the spatial discretisation). The discretisation length is represented with the constant value $\Delta z$.

The boundary conditions are given by $v_{j-1} c_{i, j-1}=$ $\dot{n}_{i, i n}$ for the first volume of discretisation $(j=1)$ and $p_{j+1}=p^{a m b}$ for the last one $\left(j=n_{\text {disc }}\right)$ as developed in [7]. From this implementation, three kind of state equations are obtained by developing (2) for the first, middle and last discretisation volumes:

$$
\dot{c}_{i, j}(t)= \begin{cases}\dot{c}_{i, j}^{A}(t), & \text { if } j=1 \\ \dot{c}_{i, j}^{B}(t), & \text { if } 2 \leq j \leq n_{d i s c}-1, \\ \dot{c}_{i, j}^{C}(t), & \text { if } j=n_{d i s c}\end{cases}
$$

where

$\dot{c}_{i, j}^{A}(t)=\frac{\dot{n}_{i, i n}(t)}{\Delta z}-K_{1} \Phi(j)-\Lambda(t)$

$\dot{c}_{i, j}^{B}(t)=K_{1}(\Phi(j-1)-\Phi(j))-\Lambda(t)$

$\dot{c}_{i, j}^{C}(t)=K_{1}\left(\Phi(j-1)-c_{i, j}(t) \sum_{i} c_{i, j}(t)\right)+K_{2} c_{i, j}(t)-\Lambda(t)$

and with

$$
\begin{aligned}
\Phi(j) & =c_{i, j}(t)\left(\sum_{i} c_{i, j}(t)-\sum_{i} c_{i, j+1}(t)\right) \\
\Phi(j-1) & =c_{i, j-1}(t)\left(\sum_{i} c_{i, j-1}(t)-\sum_{i} c_{i, j}(t)\right) \\
\Lambda(t) & =\frac{\dot{n}_{i, j}(t)}{\delta}
\end{aligned}
$$

Moreover, $K_{1}=K R T / \Delta z^{2}, K_{2}=K p^{a m b} / \Delta z^{2}$. These spatially-discretised equations will be used for the design of the NMPC controller afterwards (see 


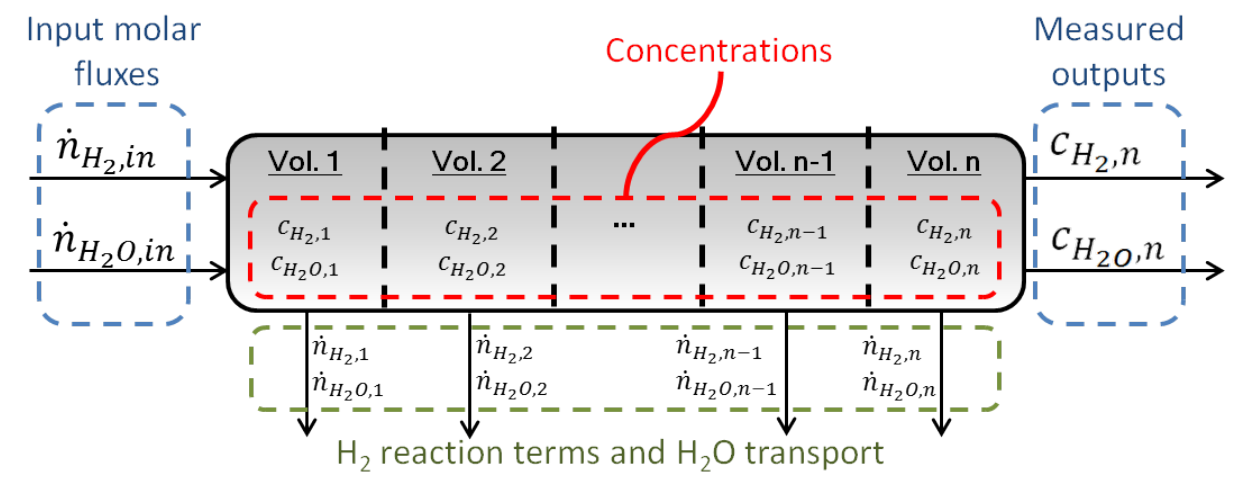

Figure 2: Generalised model of the discretised anode gas channel

\section{Section 4).}

$n_{i, i n}$ are the inlet molar flows for both $\mathrm{H}_{2}$ and $\mathrm{H}_{2} \mathrm{O}$ are considered the available manipulated variables. $n_{i, j}$ are the molar flow densities for the $\mathrm{H}_{2}$ reaction rate and the water transport through the membrane. For the simulation model considered in this paper, the initial $\mathrm{H}_{2}$ reaction rate per each of the discretised volumes is extracted from [17], where a complete electrochemical model developed from [7] is implemented. The current demanded from the load directly affects these reaction rates. Since in this paper only the anode gas channel is considered, the dynamic values of the reaction rates are assumed to follow the initial profile obtained from [17], proportionally affected by the current multiplier in Figure 5.

Similar assumptions are considered regarding the flux of water through the membrane. Since only the anode gas channel is included in this paper, starting from a nominal profile calculated with [17] model, it is assumed that the water flux will also increment proportionally with the current multiplier of Figure 5 due to the drag effect that protons create over the water molecules along the direction of the membrane. Other important model assumptions are the isothermal condition and the consideration that no liquid water formation can appear in the system. Moreover, the controller operates under output feedback of the $\mathrm{H}_{2}$ and $\mathrm{H}_{2} \mathrm{O}$ concentrations in the last discretisation volume. To infer the $\mathrm{H}_{2} \mathrm{O}$ concentration value in the last discretisation volume, a relative humidity sensor can be employed. Knowing the water saturation pressure at the system temperature and the relative humidity, it is possible to obtain the water concentration value from the water partial pressure $\left(p_{\text {out }, H_{2} O}\right)$. Moreover, knowing the output pressure $\left(p_{\text {out }}\right)$ at the end of the gas channel is a common assumption in PEMFC systems. Knowing these two pressures and since

$$
p_{\text {out }}(t)=p_{\text {out }, H_{2}}(t)+p_{\text {out }, H_{2} O}(t),
$$

the partial pressure of $\mathrm{H}_{2}\left(p_{\text {out } H_{2}}\right)$ is obtained. Finally, the hydrogen concentration is derived using the previous computed variables and (2c).

\section{NMPC Controller Design}

\subsection{Control-oriented model}

The NMPC controller introduces a prediction model similar to the one developed for the plant. The main difference is that in the prediction model the $\mathrm{H}_{2}$ reaction rate and the water transport through the membrane are considered as unknown and unmeasured disturbances while in the simulation model they are not (see Figure 4). The divergence between the plant and the prediction model is covered by the intrinsic robustness of the NMPC controller and its reconfigurability capabilities. Moreover, the statefeedback topology allows to minimize the mismatching between those two models.

From (2), the following discrete-time dynamic model is obtained:

$$
\begin{aligned}
x_{i, j}(k+1) & =x_{i, j}(k)+\left[\frac{\alpha_{j-1}(k)-\alpha_{j}(k)}{\Delta z}-d(k)\right], \\
v_{j}(k) & =\frac{K}{\Delta z}\left(p_{j}(k)-p_{j+1}(k)\right), \\
p_{j}(k) & =R T \sum_{i} x_{i, j}(k),
\end{aligned}
$$

with $\alpha_{j}(k)=v_{j}(k) x_{i, j}(k) \Delta t$ and $d(k)=\dot{n}_{i, j}(k) \Delta t / \delta$. The state variables are $x_{H_{2}, j}$, the $\mathrm{H}_{2}$ concentration, and $x_{H_{2} O, j}$, the $\mathrm{H}_{2} \mathrm{O}$ concentration, both along the $j$ volumes of the channel. Moreover, the control inputs are $u_{H_{2}} \triangleq \dot{n}_{H_{2}, \text { in }}$, corresponding to the $\mathrm{H}_{2}$ molar inflow, and $u_{\mathrm{H}_{2} \mathrm{O}} \triangleq \dot{n}_{\mathrm{H}_{2} \mathrm{O} \text {,in }}$, which denotes the water vapour molar inflow. These control inputs arise from the expansion of (6) into a volume-dependant expression as developed in (3). Here, $\Delta t$ is the sampling time that, for this case study, is $10 \mathrm{~ms}$ and $k \in \mathbb{Z}$ denotes the discrete-time variable.

\subsection{State observer}

Since the system construction is enclosed, there is no information about the values of all the states. For this reason, a state observer is implemented using a measure of the output concentrations of the channel 
in order to reconstruct the entire state vector. The topology of the observer is based on a classical Luenberger structure [18]. Making use of the nomenclature proposed in this paper, the observer equations can be written as

$$
\begin{aligned}
\hat{x}_{i, j}(k+1) & =A \hat{x}_{i, j}(k)+L\left[y_{i}(k)-\hat{y}_{i}(k)\right] B u_{i}(k), \\
\hat{y}_{i}(k) & =C \hat{x}_{i, j}(k)+D u_{i}(k),
\end{aligned}
$$

where $\hat{x}$ and $\hat{y}$ denotes the estimated states and estimated output respectively and $A, B, C$ and $D$ are the system matrices of suitable dimensions. The output concentrations are denoted by $y$. The model matrix $A$ is computed from the linearisation of the model equations (2) around a given operating point. Since the focus of this paper is to regulate the gas concentrations, the dynamical divergence that appears from using a linear observer versus a nonlinear one can be accepted under the premise that the states must always be close to the initial operating point.

Following [18], and considering the observer (7), the observer error, namely $\bar{e}$, satisfies

$$
\bar{e}_{i}(k+1)=(A-L C) \bar{e}(k),
$$

where $L$ is the observer gain matrix.

For the discretised system considered in this paper, the $L$ matrix is designed in such a way that the poles $(A-L C)$ lie inside the unit circle (Schur matrix). Moreover, a general rule when using Luenberger observers is to design the observer poles between 4 and 10 times faster than the slowest dynamics from the linearised system, being this the criteria employed in this paper.

\subsection{Spatial discretisation}

As presented in (3), the mass balance equations are discretised using finite differences. While the discretisation of the partial differential equations allows to make use of lumped systems theory and to take advantage of the known boundary conditions, it introduces a high computational effort.

A fine discretisation would be the ideal case. Nevertheless, the computational effort when increasing the number of discretisation volumes leads to determine the suitable trade-off between discretisation detail and computational burden. For this paper, the number of discretisation volumes has been selected in order to guarantee the observability condition, which has only been proved to hold up to 9 volumes. For more volumes, the observer is not able to reconstruct the full-state vector due to numerical problems and thus, the implemented output-feedback NMPC controller is no longer feasible. As a result of this sensitivity analysis, simulations have been carried out considering 9 discretisation volumes, which implies models (2) or (6) with 18 concentration values (system states).

\subsection{Control objectives}

The degradation and life expectancy of the PEMFC is directly related to the behaviour of the internal variables of the system. As stated, this paper focuses on the control of the concentration values along the anode gas channel. To guarantee an optimal performance and reduce the degradation that could diminish the life expectancy of the system, the control objectives for this work are straightforward: it is needed to maintain certain values of $\mathrm{H}_{2}$ and $\mathrm{H}_{2} \mathrm{O}$ concentrations $\left(x_{\mathrm{H}_{2}}^{r e f}\right.$ and $\left.x_{\mathrm{H}_{2} \mathrm{O}}^{r e f}\right)$ along all the channel no matter what PEMFC reaction consumption profile is given.

Indeed, a PEMFC-based system is subject to variations in the demanded current, which affect the reaction terms and the water transport through the system. Nevertheless, the control strategy aims to maintain a given operation condition for which the system is known to perform adequately, reducing the effect of the disturbances. The reaction terms and water transport are considered unmeasurable disturbances for the NMPC controller as stated in Section 4.1. The global control problem can also be defined as a disturbance rejection problem.

The $\mathrm{H}_{2}$ consumption is related to the PEMFC output voltage and thus, the generated power of the system. This comes dictated by the current demand of the load connected to the PEMFC-based system. When the $\mathrm{H}_{2}$ consumption varies due to a current change, the hydrogen concentration profiles fluctuate, and therefore this can influence the quality of the chemical reaction. Besides, it is important to properly hydrate the membrane (but not too much to avoid liquid water formation) to guarantee the suitable performance of the overall system. Concentrations $x_{\mathrm{H}_{2}}^{r e f}$ and $x_{\mathrm{H}_{2} \mathrm{O}}^{r e f}$ are the reference values for which the designed NMPC controller should steer the system to its stationary point in finite time, in spite of the disturbance profile $\dot{n}_{i, j}$ given by the electrical consumption of the load, which is also unknown beforehand.

\subsection{Control volumes}

Taking advantage of the finite-element discretisation of the model (3), the implementation of the control strategy includes the possibility of considering different discretised volumes to perform the regulation of the concentrations in the gas channel. Indeed, the designed controller allows to select one or two discretised volumes from the $n_{\text {disc }}$. This selection is limited due to the increasing complexity of adding 
more controlled volumes to the optimisation problem. Selecting two controlled volumes it is possible to choose different combinations from the discretised anode gas channel (see Figure 2).

The possibility of choosing between numerous combinations of control volumes allows to study different simulation conditions in order to determine the best choice between different control configurations. This matter will be deeply analysed in Section 5 .

\subsection{System constraints}

This paper sets up the theoretical basis to a future implementation of the proposed NMPC controller in a real PEMFC-based system. The values of the constraints are obtained taking into account nominal values from models reported in the literature $[7,17]$ and considering some percentage of tolerance to simulate the controller. Nevertheless, the control algorithm is flexible to introduce the real values, which can be extracted from the information given by the manufacturer of the given system.

The input constraints depend mainly on the equipment employed to inject both the hydrogen and water molar flows into the channel. Here they are set as

$$
\begin{gathered}
0 \leq u_{H_{2}} \leq 40 \mathrm{~mol} \mathrm{~m}^{-2} \mathrm{~s}^{-1} \\
0 \leq u_{H_{2} O} \leq 15 \mathrm{~mol} \mathrm{~m} \mathrm{~m}^{-2} \mathrm{~s}^{-1} .
\end{gathered}
$$

The input steady-state values are used to obtain the initial operation condition for which the simulation starts, i.e.,

$$
u_{H_{2}}^{\mathrm{ss}}=35 \mathrm{~mol} \mathrm{~m}^{-2} \mathrm{~s}^{-1}, \quad u_{H_{2} O}^{\mathrm{ss}}=10 \mathrm{~mol} \mathrm{~m}^{-2} \mathrm{~s}^{-1} .
$$

This initial operation condition determines the $\mathrm{H}_{2}$ and $\mathrm{H}_{2} \mathrm{O}$ concentrations to be the set-point values for which the NMPC controller aims to regulate the system.

Apart from the input constraints, there is another set of hard constraints related to the admissible concentration values throughout the channel, not only at the controlled volumes. These constraints are

$$
\begin{aligned}
& 0<x_{H_{2}, j} \leq 20 \mathrm{~mol} \mathrm{~m}^{-3}, \quad \forall j, \\
& 0<x_{H_{2} O, j} \leq 4 \mathrm{~mol} \mathrm{~m} \mathrm{~m}^{-3}, \quad \forall j,
\end{aligned}
$$

where (10a) is related to the hydrogen concentrations. Since all the volumes are constrained to have non-zero and positive concentration values, there will always be enough $\mathrm{H}_{2}$ in the anode to satisfy the power demanded by the load due to its variations within normal operation conditions. However, it is possible that, if the power demand is enough to saturate the $\mathrm{H}_{2}$ molar inflow, the system enters in starvation, a situation that would result in a failure to meet constraint (10a) in one or more of the discretised volumes. Constraint (10b) is related to the water concentration along the channel, affecting the humidification of the membrane, which has to be suitable to operate the system within proper conditions of conductivity and degradation.

\subsection{Cost function}

Given the control objectives stated for this problem, the resultant cost function for the controlled volumes $\left(n_{\text {cont }}\right)$ can be expressed as

$$
J(K)=\sum_{j=0}^{n_{\text {cont }}}\left\|x_{j}-x_{j}^{r e f}\right\|_{W_{x}}^{2}+\|\Delta u\|_{W_{u}}^{2},
$$

where $x_{j}=\left[x_{H_{2}, j}, x_{H_{2} O, j}\right]^{T}$ and $\Delta u=$ $\left[\Delta u_{H_{2}}, \Delta u_{H_{2} O}\right]^{T}$ with the slew rate $\Delta u_{i}(k) \triangleq$ $u_{i}(k)-u_{i}(k-1)$. Similarly, $x_{j}^{\mathrm{ref}}=\left[x_{H_{2}, j}^{\mathrm{ref}}, x_{H_{2} O, j}^{\mathrm{ref}}\right]^{T}$. Moreover, notation $\|\cdot\|_{W}^{2}$ indicates the quadratic weighted norm, where the weighting matrices are defined as $W_{x}=\operatorname{diag}\left(\gamma_{x_{1}} ; \gamma_{x_{2}}\right)$ and $W_{u}=\gamma_{u} \mathbf{I}$, with $\gamma_{*} \in \mathbb{R}$ and $\mathbf{I}$ being an identity matrix of suitable dimensions. Matrices $W$ allow to prioritise each control objective within the cost function (11). Notice that, apart from the state error, minimisation terms for the slew rate of the two manipulable inputs have been included in (11), avoiding abrupt changes in the control inputs that could damage the system devices.

The optimal tuning of the weighting matrices is out of the scope of this paper. However, a standard trialand-error procedure based on several simulation has allowed to define a suitable combination of weighting matrices such as the prioritisation of the control objectives were achieved. The effect of different combination of weights over the mean absolute error (MAE) of the state variables, which is defined as a performance indicator of the system and further discussed in Section 5.3, is presented in Figure 3. In this figure, it can be seen how the system error oscillates (hence the states) when tracking errors for both $\mathrm{H}_{2}$ and $\mathrm{H}_{2} \mathrm{O}$ are equally prioritised. On the other hand, lower rates between $\gamma_{x_{1}}$ and $\gamma_{u}$ imply higher MAE values. The selected set of parameters is reported in Table 2 .

\subsection{NMPC Design}

The algorithm employed for the disturbance rejection approach has been taken from [19], which has been adopted for the proposed case study. The parameters of the algorithm and their units can be found in Table 2. Therefore, the design of the NMPC controller for the proposed case study in this paper is based on Problem 1. 


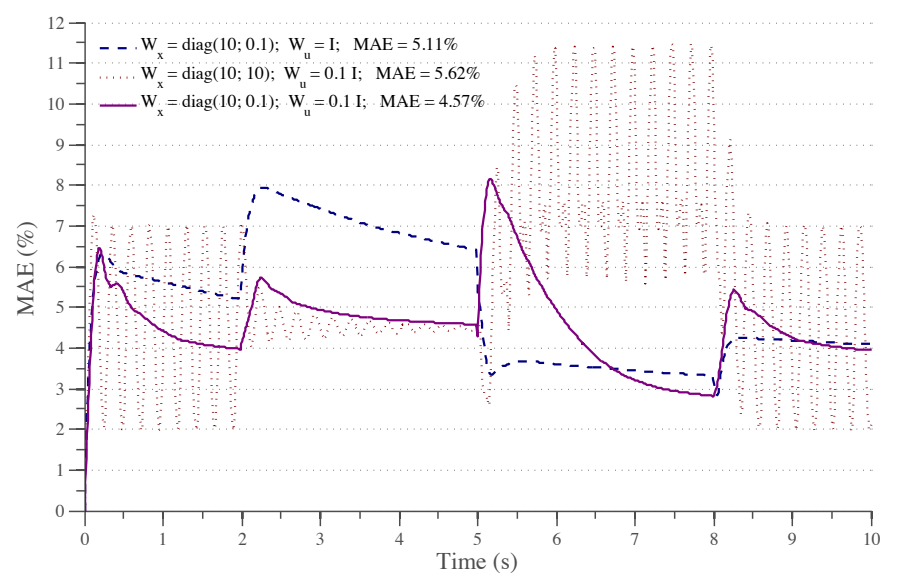

Figure 3: MAE behaviour for different values of weighting matrices (MPC tuning)

Table 2: NMPC setup parameters

\begin{tabular}{lll}
\hline parameter & description & value \\
\hline$\gamma_{x_{1}}$ & $\mathrm{H}_{2}$ error penalisation & 10 \\
$\gamma_{x_{2}}$ & $\mathrm{H}_{2} \mathrm{O}$ error penalisation & 0.1 \\
$\gamma_{u}$ & inputs slew rate penalisation & 0.1 \\
$H_{p}$ & prediction horizon & 5 \\
$H_{c}$ & control horizon & 5 \\
$\Delta t$ & sampling time & $10 \mathrm{~ms}$ \\
$T_{\text {sim }}$ & simulation time & $10 \mathrm{~s}$ \\
\hline
\end{tabular}

Problem 1 (NMPC Design). Let $^{1}$

$$
\mathbf{u}(k) \triangleq\left(u(0 \mid k), \ldots, u\left(H_{p}-1 \mid k\right)\right)
$$

be the sequence of control inputs over a fixed-time prediction horizon $H_{p}$, depending also on the initial condition $x(0 \mid k) \triangleq x_{0}$. Hence, the NMPC design is based on the solution of the finite-time open-loop optimization problem (FTOOP)

$$
\min _{\mathbf{u}(k) \in \mathbb{R}^{m H_{p}}} J\left(x_{0}, \mathbf{u}(k)\right),
$$

subject to

- system model in (6) over $H_{p}$,

- input constraints in (9) over $H_{p}$,

- state constraints in (10) over $H_{p}$,

where $J(\cdot): \mathbb{U}^{m H_{p}} \times \mathbb{R}^{H_{p}} \mapsto \mathbb{R}$ in (13) is the cost function, with $m=2$ and $H_{p}=H_{u}$, where $H_{u}$ denotes the control horizon. Assuming that the FTOOP (13)

\footnotetext{
${ }^{1}$ Here, $f(k+i \mid k)$ denotes the prediction of the variable $f$ at time $k+i$ performed at $k$. For instance, $x(k+i \mid k)$ denotes the prediction of the system state, starting from its initial condition $x(0 \mid k)=x(k)$.
}

is feasible, there will be an optimal solution for the sequence of control inputs

$$
\mathbf{u}^{*}(k) \triangleq\left(u^{*}(0 \mid k), u^{*}(1 \mid k), \ldots, u^{*}\left(H_{p}-1 \mid k\right)\right)
$$

and then, according to the receding horizon philosophy, $u_{i}^{*}(0 \mid k)$ is applied to the system, while the process is repeated for the next time instant $k \in \mathbb{Z}$.

The overall topology for the controller implementation is shown in Figure 4.

\section{Simulation Results}

The initial state vector for all simulations is $x_{0}$ $=(24.47,7.09,22.73,6.69,20.89,6.27,18.94,5.80$, $16.85,5.29,14.57,4.71,12.05,4.02,9.14,3.17,5.53$, $2.01)^{T}$, in $\mathrm{mol} \mathrm{m} \mathrm{m}^{-3}$. The simulations have been carried out using fmincon function in MATLAB ${ }^{\circledR}$ R2011a (32 bits), running in a PC Intel ${ }^{\circledR}$ Core $^{\mathrm{TM}}$ i7 $740 \mathrm{QM}$ at $1.73 \mathrm{GHz}$ with $4 \mathrm{~GB}$ of RAM.

\subsection{Simulation scenario}

The simulation scenario starts with the system working in an operating point with $\mathrm{H}_{2}$ reaction and $\mathrm{H}_{2} \mathrm{O}$ transport terms unknown by the controller. As shown in Figures 2 and 4, the output concentration values are feed into the state observer (besides the manipulable variables from the controller) and the estimated full-state vector is given to the NMPC controller, which computes the most suitable sequence of control actions at each time step $\Delta t$.

There exists a direct relation between the electrical current demanded by the load and the $\mathrm{H}_{2}$ reaction rates of the PEMFC [20]. To study the performance of the NMPC controller, a given current profile is demanded from the system at each time instant. The current consumption multiplier for each of the discretised volumes of the system is shown in Figure 5.

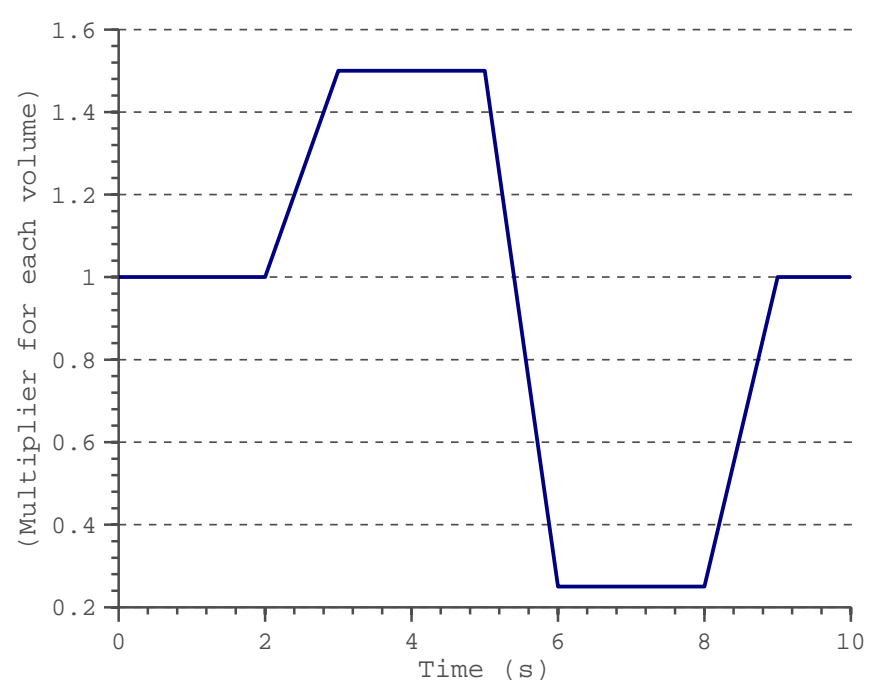

Figure 5: Current consumption profile multiplier 


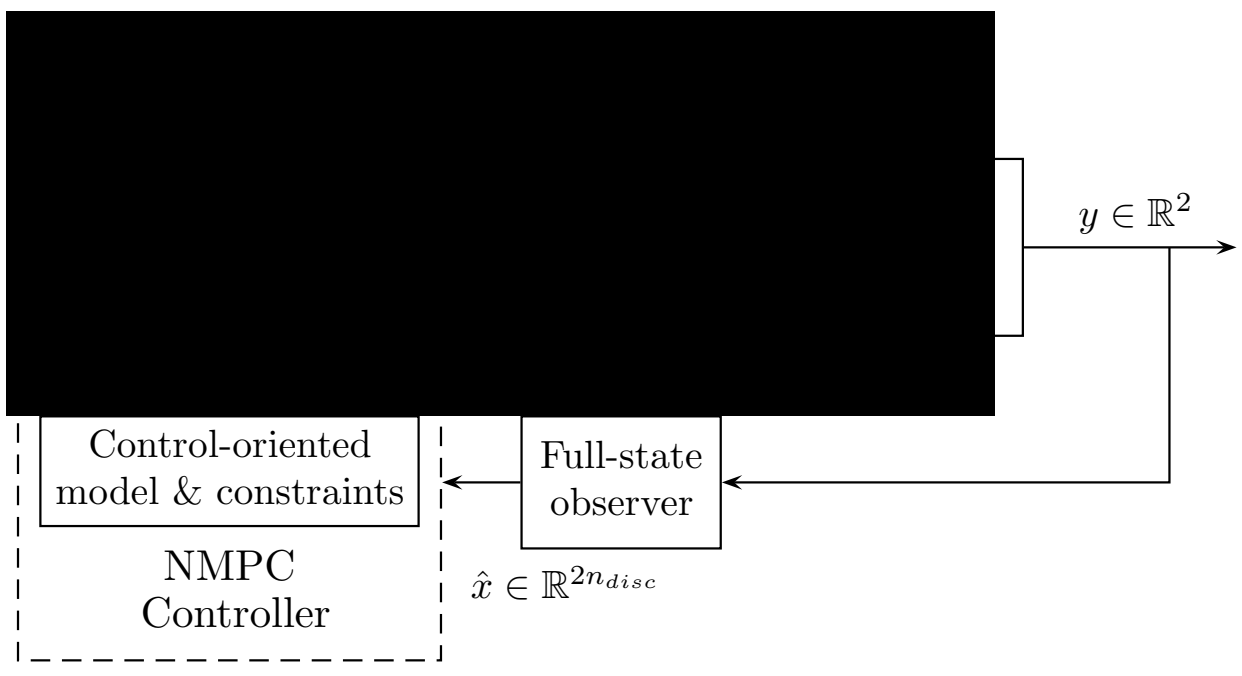

Figure 4: Closed-loop control topology of the case study

Different combinations of controlled volumes are simulated and analysed in subsequent sections in order to determine which one is the most appropriate controller configuration for the desired dynamical behaviour of the case study.

\subsection{Controller setup}

As stated in the introduction, one of the main capabilities of the NMPC algorithms is their vast reconfigurability via the tuning of its parameters, such as prediction and control horizons, penalisation terms, norms, etc. Table 2 shows the controller setup parameters and the sampling and simulation times of each one of the simulation scenarios.

\subsection{Performance indicator}

The regulation error through the channel (e) is defined as the difference between the desired state vector $\mathbf{x}^{\text {ref }}$ and the current value of the states $\mathbf{x}$. When the discretisation is performed, this difference is expanded to all the finite elements in the model. To study and compare the forthcoming simulation results, a performance indicator is introduced. For this paper, the mean absolute error (MAE)

$$
M A E=\frac{1}{n_{\text {disc }}} \sum_{p=1}^{n_{\text {disc }}}\left|e_{p}\right|=\frac{1}{n_{\text {disc }}} \sum_{p=1}^{n_{\text {disc }}}\left|\mathbf{x}_{p}-\mathbf{x}_{p}^{r e f}\right|
$$

is proposed. Note that (15) is defined for the discretised model presented in (3), which considers all the $n_{\text {disc }}$ volumes in the anode gas channel, not only at control ones. In (15), subscript $p$ refers to the $p$-th element of the state vector $\mathbf{x}$. As said, this error is computed by comparing the state values at a given moment of the simulation with their reference values, which in a regulation problem, is a constant value. Particularly, the desired reference values are the initial conditions $\mathbf{x}_{\mathbf{0}}$ of the plant formerly defined. Therefore (15) can be expressed as

$$
M A E=\frac{1}{n_{\text {disc }}} \sum_{p=1}^{n_{\text {disc }}}\left|\mathbf{x}_{p}-\mathbf{x}_{0, p}\right| .
$$

\subsection{Results and discussion}

As introduced in Section 4, the cost function (11) and the implementation of the problem are designed to allow different combinations of control volumes in the discretised anode gas channel. In Table 3, the MAEs over all the simulation time are presented for six possible combinations of control volumes to achieve the desired regulation along the channel.

Table 3: Mean Absolute Error of the simulations

\begin{tabular}{llc}
\hline control volume(s) & acronym & average MAE (\%) \\
\hline first & $(\mathrm{F})_{V}$ & 6.26 \\
first-middle & $(\mathrm{F}-\mathrm{M})_{V}$ & 5.24 \\
middle & $(\mathrm{M})_{V}$ & 4.57 \\
middle-last & $(\mathrm{M}-\mathrm{L})_{V}$ & 4.67 \\
last & $(\mathrm{L})_{V}$ & 8.03 \\
first-last & $(\mathrm{F}-\mathrm{L})_{V}$ & 5.55 \\
\hline
\end{tabular}

The behaviour of the MAE for all the different scenarios of control volumes combinations is represented in Figure 6. As it can be extracted from the figure, the disturbance rejection capability is similar for most of the cases with some exceptions, like when only the first and last volumes are considered in the cost function.

From the average MAE and the results presented in Table 3, it can be concluded that the best performance is obtained with the configuration that has the middle volume as the controlled volume $\left(\right.$ the $(\mathrm{M})_{\mathrm{V}}$ 


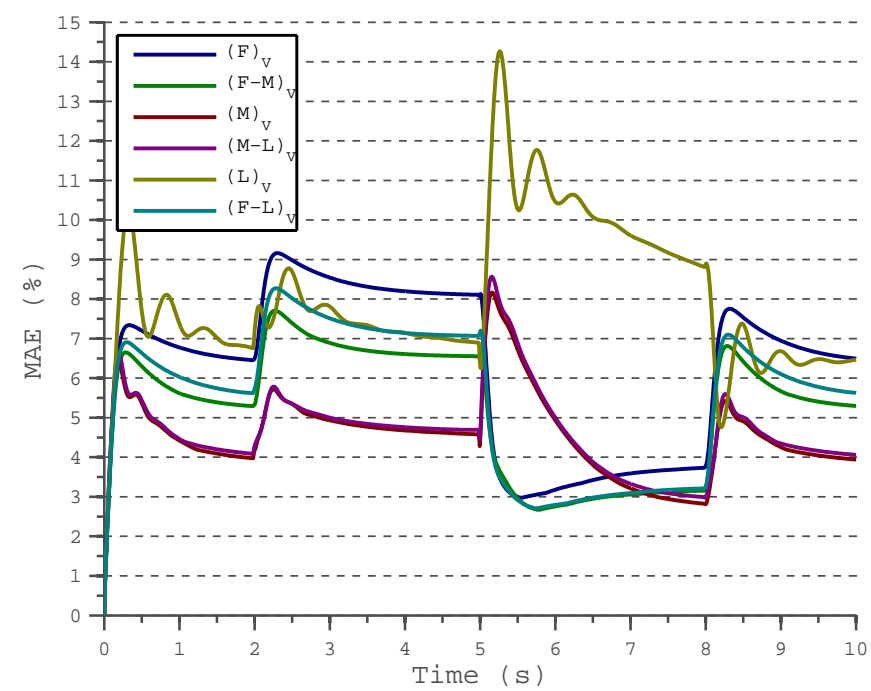

Figure 6: MAE profile for six control volumes combinations

case). This is due to the lower average MEA of the disturbance rejection problem and the lower overshoots of the error for this combination. Also, notice that the full regulation of two volumes in steady state is not possible because there are not enough degrees of freedom with only two manipulated inputs.

The dynamical behaviour of the controlled outputs and the manipulated inputs applied to the system for the $(\mathrm{M})_{\mathrm{V}}$ case are shown in Figure 7, where subscript $v c$ denotes the state values of the controlled volume.

It is possible to plot the whole range of concentrations profile during the duration of the simulation process. This is presented in Figure 8 and shows that, with the proposed control strategy based on NMPC, the concentrations remain in a narrow and bounded range of values, which guarantee an stable behaviour of the energy system no matter the load current variation (and thus, reaction disturbances).

\subsection{Observer performance}

The linear state observer feeds the NMPC controller with the estimated state vector as described in Section 4. Figure 9 shows the behaviour of the estimator when the controlled states shown in Figure 7 a move away from the initial operating point as a consequence of the current multiplier proposed in Figure 5. As expected, the observed states $(\hat{x})$ present a different behaviour compared to the system states from the simulation model.

In Figure 9a, which refers to the $\mathrm{H}_{2}$ concentration estimation, the divergence appears more clearly when there is a change in the current multiplier, which drives away the state from its valid region according to the observer model (e.g. at times 2 and $5 \mathrm{~s}$ ). However, these errors can be considered negligible compared to the overall magnitude of the states. On the other hand, in Figure 9b, wich refers to the water vapour concentration estimation, the difference

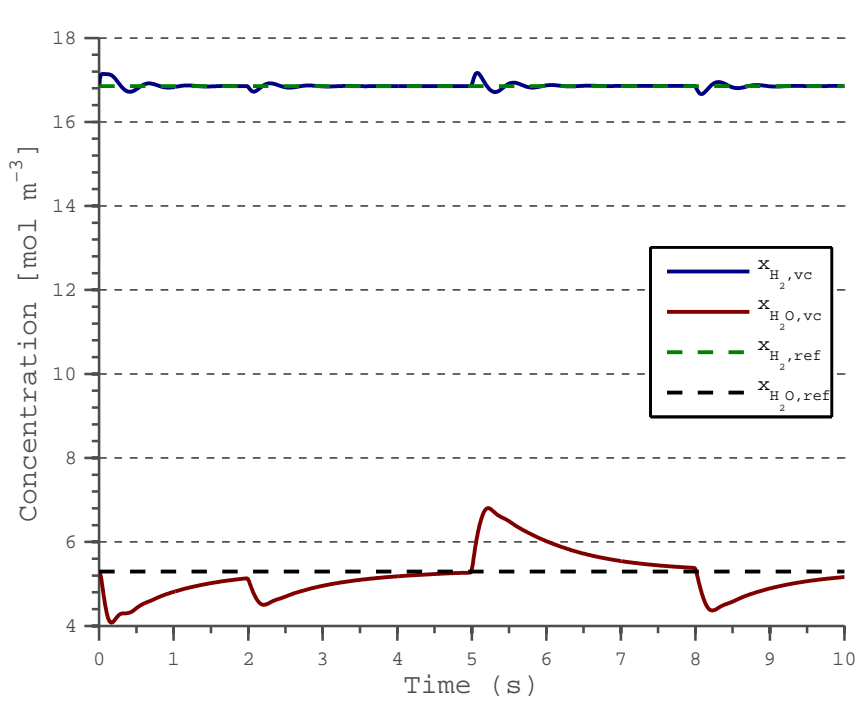

(a) Controlled outputs

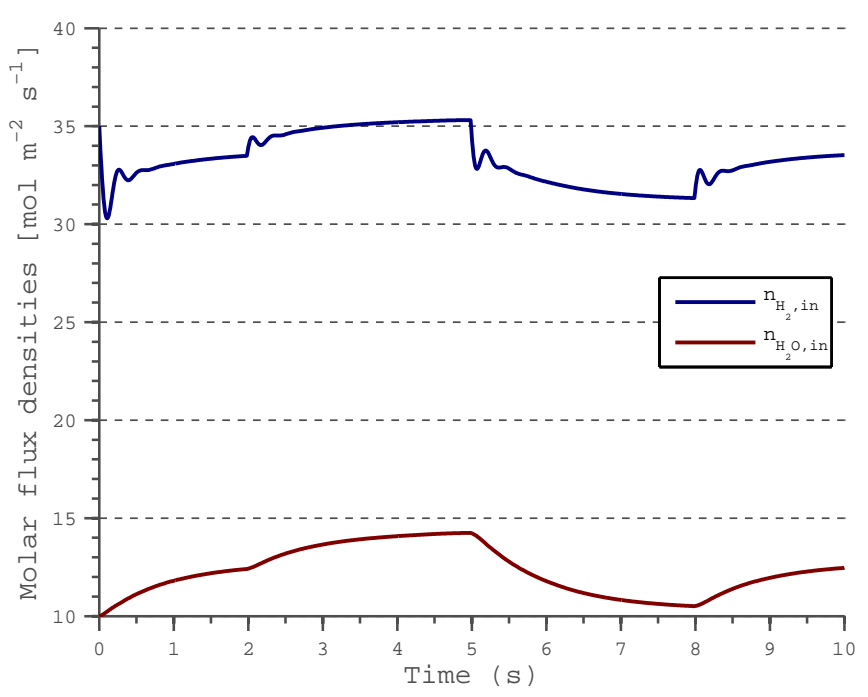

(b) Manipulable inputs

Figure 7: Input and output dynamical behaviour with the NMPC-based closed loop

between the observed state and the plant state is seen to be significant at a dynamical level, having the observed state a slower response compared to the plant behaviour. Nevertheless, the observed state approaches converges to the plant response when the response approaches its equilibrium condition.

\section{Conclusions}

In this paper, an NMPC controller design has been designed to regulate the concentration profiles in a PEMFC anode gas channel. This strategy allows to handle the nonlinear dynamics of the plant in order to maintain a given operating condition and to avoid the degradation of the system. The performance of the controller has been evaluated, obtaining satisfactory results for a given simulation scenario. The controller is robust enough to reduce the effect of the unknown disturbances that influence the system when a change occurs in the load. 


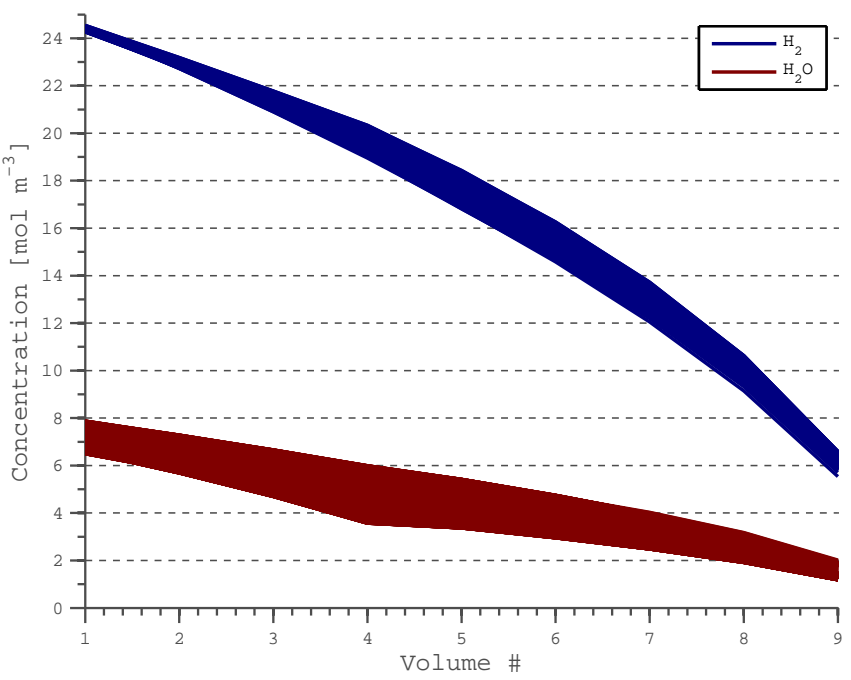

Figure 8: Concentrations distribution through all the discretisation volumes along the simulation scenario

The MAE along the anode (see Figure 6) depends on the controlled volumes selected as it has been shown with the analysis of the obtained results. Indeed, when stating the cost function, the selection of certain combinations of controlled volumes is more convenient from the performance point of view. Specifically, the inclusion of two control volumes in the cost function reduces in general the regulation error. However, controlling only the middle volume shows to be the most suitable option for this problem. With the proposed control strategy, the variation of the concentrations are guaranteed to be limited to small values along the channel, thus achieving the desired regulation even in the presence of unknown disturbances. An impending improvement of the proposed approach is to increase the number of controlled volumes included in the cost function. Although, it must be remarked that there is not enough degrees of freedom in the problem to achieve perfect regulation of all the volumes, nonetheless the increasing of the number of control volumes can improve the overall optimisation problem results.

The number of discretisation volumes is limited by the design of the observer. Future research will be aimed at generalising the approach to higher number of volumes in order to increase the detail of the solution. In addition, this paper presents the linear estimation of the states from the measured outputs of a nonlinear plant. The observation error is the main source of error of the control strategy presented in this paper. However, it is forthcoming to include the nonlinear state observer approach to improve the full-state vector estimation and reduce the regulation error with more precise information about the behaviour of the entire plant.

Only an anode gas channel of a PEMFC is studied in this paper. Taking into account that the re-

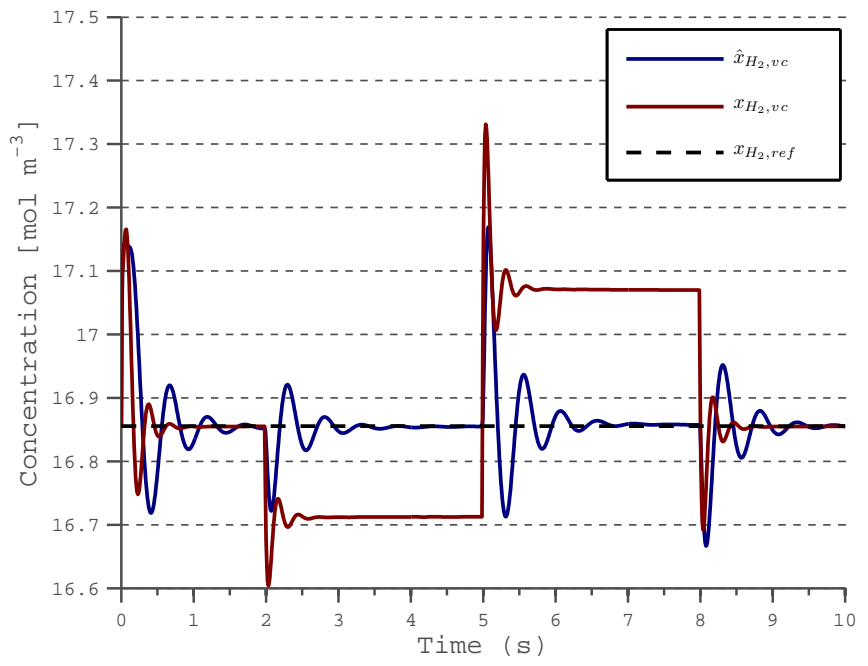

(a) $\mathrm{H}_{2}$ estimation

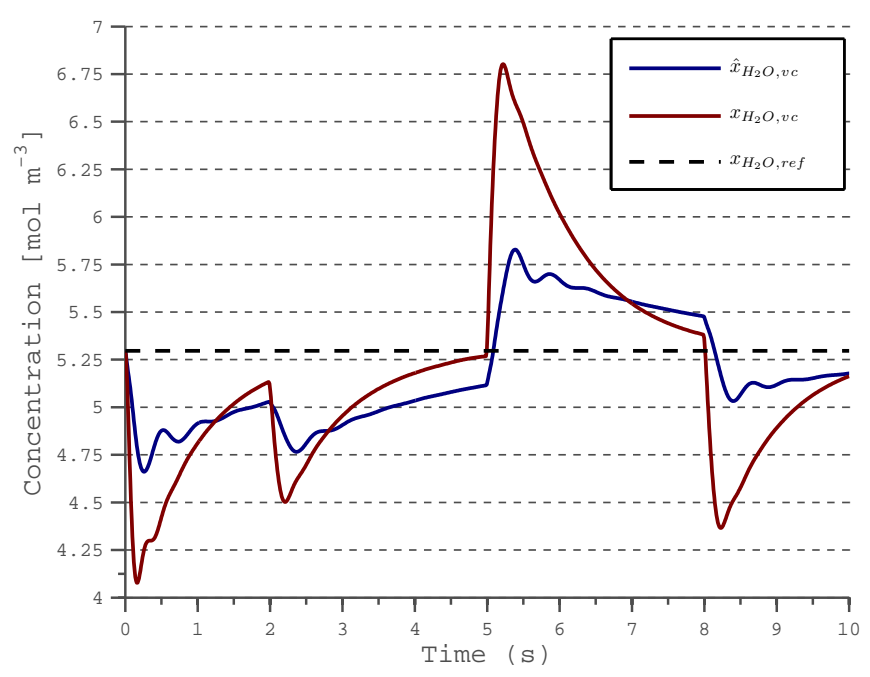

(b) $\mathrm{H}_{2} \mathrm{O}$ estimation

Figure 9: Behaviour of the linear state estimator versus the plant states in the control volume

action and water transport terms are considered as unknown and unmeasurable disturbances, the proposed solution is immediately applicable to the cathode gas channel since the modelling equations for the gas concentration behaviours are equivalent to those that model the anode gas channel. The next step in the research line is to include all PEMFC components (e.g., cathode, membrane, gas diffusion layers and gas catalyst layers) in order to implement the proposed controller to the entire PEMFC-based system, even over a real test-bench.

\section{Acknowledgements}

This work has been partially funded by the Spanish national project MESPEM (Ref. DPI2011-25649) and the MACPERCON project (Ref. 201250E027) of the Spanish National Research Council (CSIC). 


\section{References}

[1] J. T. Pukrushpan, A. G. Stefanopoulou, H. Peng, Control of fuel cell power systems: principles, modeling, analysis and feedback design, Springer, 2004.

[2] P. H. Rodatz, Dynamics of the polymer electrolyte fuel cell: experiments and model-based analysis, Ph.D. thesis, Swiss Federal Institute of Technology (2003).

[3] F. Bianchi, C. Ocampo-Martinez, C. Kunusch, R. Sanchez-Pena, Fault-tolerant unfalsified control for pem fuel cell systems, IEEE Transactions on Energy ConversionIn press.

[4] M. Bavarian, M. Soroush, I. G. Kevrekidis, J. B. Benziger, Mathematical modeling, steady-state and dynamic behavior, and control of fuel cells: A review, Industrial \& engineering chemistry research 49 (17) (2010) 7922-7950.

[5] K. K. T. Thanapalan, G. P. Liu, J. G. Williams, B. Wang, Review and analysis of fuel cell system modelling and control, International Journal of Computer Aided Engineering and Technology 1 (2) (2009) 145-157.

[6] R. N. Methekar, V. Prasad, R. D. Gudi, Dynamic analysis and linear control strategies for proton exchange membrane fuel cell using a distributed parameter model, Journal of Power Sources 165 (1) (2007) 152-170.

[7] M. Mangold, A. Bück, R. Hanke-Rauschenbach, Passivity based control of a distributed PEM fuel cell model, Journal of Process Control 20 (3) (2010) 292-313.

[8] J. M. Maciejowski, Predictive control with constraints, Pearson education, 2002.

[9] A. Vahidi, W. Greenwell, A decentralized model predictive control approach to power management of a fuel cell-ultracapacitor hybrid, in: American Control Conference, New York, NY, USA, 2007, pp. 5431-5437.

[10] C. Bordons, A. Arce, A. J. Del Real, Constrained predictive control strategies for PEM fuel cells, in: American Control Conference, Minneapolis, MN, USA, 2006, pp. 6-pp.

[11] A. Arce, D. R. Ramirez, A. J. del Real, C. Bordons, Constrained explicit predictive control strategies for PEM fuel cell systems, in: 46th IEEE Conference on Decision and Control, New Orleans, LA, USA, 2007, pp. 6088-6093.
[12] M. Fiacchini, T. Alamo, C. Albea, E. F. Camacho, Adaptive model predictive control of the hybrid dynamics of a fuel cell system, in: IEEE International Conference on Control Applications, Signapore, 2007, pp. 1420-1425.

[13] Q. Chen, S. Quan, C. Xie, Nonlinear predictive control for oxygen supply of a fuel cell system, in: International Joint Conference on Neural Networks, Atlanta, GA, USA, 2009, pp. 518-521.

[14] A. J. del Real, A. Arce, C. Bordons, Hybrid model predictive control of a two-generator power plant integrating photovoltaic panels and a fuel cell, in: 46th IEEE Conference on Decision and Control, New Orleans, LA, USA, 2007, pp. 5447-5452.

[15] J. Luna, C. Ocampo-Martinez, M. Serra, Nonlinear predictive control for the concentrations profile regulation in a PEM Fuel Cell anode gas channel, in: European Control Conference, Strasbourg, France, 2014, pp. 1807-1812.

[16] W. Neubrand, Modellbildung und simulation von elektronen membranverfahren, Ph.D. thesis, $\mathrm{PhD}$ thesis, Universitat Stuttgart (1999).

[17] M. Sarmiento-Carnevali, M. Serra, C. Batlle, Distributed parameter model simulation tool for pem fuel cells, International Journal of Hydrogen Energy 39 (8) (2014) 4044-4052.

[18] D. G. Luenberger, Observing the state of a linear system, IEEE Transactions on Military Electronics 8 (2) (1964) 74-80.

[19] L. Grüne, J. Pannek, Nonlinear model predictive control, 2011, Springer.

[20] C. H. Woo, J. B. Benziger, Pem fuel cell current regulation by fuel feed control, Chemical engineering science 62 (4) (2007) 957-968. 